\title{
Surge prevention in gas turbines: an overview over historical solutions and perspectives about the future
}

\author{
Carlo Alberto Niccolini Marmont Du Haut Champ ${ }^{1, *}$, Aristide Fausto Massardo ${ }^{1}$, Mario \\ Luigi Ferrari ${ }^{1}$, and Paolo Silvestri ${ }^{1}$ \\ ${ }^{1}$ University of Genoa, Thermochemical Power group (TPG), 16145 Genoa, Italy
}

\begin{abstract}
The aim of the present work is to retrace experimental, analytical and numerical analyses which deal with compressor instability phenomena, such as rotating stall and surge. While the first affects only the machine itself, the second involves the whole energy system. Surge onset is characterized by strong pressure and mass flow rate fluctuations which can even lead to reverse flow through the compressor. Experimental studies on prevention of axial compressor fluid dynamic instabilities, which can be propagated and eventually damage the solid structure, have been carried out by many authors. The first important studies on this topic tried to underline the main aspects of the complex detailed mechanism of surge, by replacing the compression system with an equivalent conceptual lumped parameter model. This is specially meant to capture the unsteady behaviour and the transient response of the compression system itself, particularly its dependence on variations in the volume of discharge downstream and in the settings of the throttle valve at its outlet (which simulates the actual load coupled to the compressor). Greitzer's model is still regarded as the milestone for new investigations about active control and stabilization of surge and, more generally, about active suppression of aerodynamic instabilities in turbomachinery. During the last years, a lot of simulations and experimental studies about surge have been conducted on multistage centrifugal compressors with different architectures (e.g. equipped with vaneless or vaned diffusers). Moreover, further kinds of analysis try to extend the stable working zone of compressors, identifying stall and surge precursors extractable from information contained in the vibro-acoustical and rotodynamic response of the system.
\end{abstract}

\section{Introduction}

The present energy worldwide scenario requires energy plants working far from their ondesign conditions: this causes transient operations and partial load working conditions to happen frequently. For dynamic compressors, this means they find themselves working close to their instability region (i.e. low mass flow rates), especially during load and velocity transients or emergency shutdowns [1]. Moreover, in recent years the need to

\footnotetext{
* Corresponding author: carloalberto.niccolinimarmontduhautchamp@edu.unige.it
} 
increase global efficiency of energy plants has led to the success of advanced cycles, such as fuel cells hybrid systems, based on micro Gas Turbines (mGT). This is an important issue for centrifugal compressors, which are normally employed in this kind of systems, since now they have to face higher volumes in comparison to combined cycle plants [2]. The increase of discharge volumes downstream of compression systems has always represented one of the greatest matters difficult to be solved, related to turbomachinery and energy systems. From the first applications of Gas Turbines technology, e.g. in military aviation, engineers and researchers have always struggled with the instabilities which affect axial compressors during operating conditions, trying to prevent them to avoid machine operation in its unsafe region. The main expressions of instability of compression systems are rotating stall and surge, which respectively involve the compressor itself and the whole energy system in which the machine is placed.

Rotating stall is a disturbance of the flow which takes place along the circumferential coordinate of the machine and can propagate from one inter-blade channel to another, leading to a performance decrease, but still not comprising safety operating of the system. Its onset can be detected by pressure measurements which reveal a distortion in the ondesign expected load distribution along the blade surface in a stage of a certain row of the machine. Surge is not anymore only a disturbance, but it represents, to all effects, an anomalous and dangerous operating condition of a compression system [3].

The first attempts to describe these two related physical phenomena were essentially experimental, as for example can be seen in [4], where investigations on multi-stage compressors were performed to study the stall margin decrease that takes place if an aeroengine accelerates; the authors noticed and described for firsts the change in stage load that happens in acceleration transients. Experimental analysis techniques were at that time prevalent because until recent years ago numerical techniques had still to developed (the Finite Volume Method, largely used in turbomachinery thermo-fluid-dynamic studies, was implemented in the '80). Starting from lots of experimental campaigns conducted on compressors of different architectures, analytical models to predict stall and surge onset, and then even to correct the operating condition of compression systems were developed. Attempts to achieve active control systems for big Gas Turbine plants were made by several authors: these were essentially based on operating on opening valves and variable volumes when the compressor entered its stability region limit.

The present paper will take a look to some of these analyses conducted in the past, highlighting the ideas behind each proposed solution to face this issue, and then will analyse more in detail techniques nowadays used in trying to solve the same problem. Large importance will be given to vibro-acoustic analyses and digital processing of physical signals acquired from probes and sensors, trying to exploit and extract all information contained and possibly hidden in original signals [5]. Another relevant kind of analysis is the one which considers also the mechanical behaviour of compressors, both on the force measurements side [6] and on the rotodynamic modelling point of view [7], capturing information on fluid-dynamic phenomena that are taking place inside the machine, exploiting possible coupled response of complex systems.

\section{Initial descriptions of stall and surge}

As reported in [8], the very initial studies made important observations on the physical phenomena of stall and surge, even if at that time no one was able to distinguish between them. It is to be underlined that in [9], the authors had already made measurements with optical pressure probes and were able to prove the influence of duct volumes on surge onset: this evidence will be formalized only 35 years later by Greitzer's analytical model. 


\subsection{Experimental characterization}

Lots of studies were carried out in the past, trying to reduce the instability working region of dynamic compressors, starting from axial machines and then also on radial ones, even multi-stage, till to experimental campaigns about axial-centrifugal compressors, as reported in [3]. A remarkable example of experimental analysis on multistage centrifugal compressor can be found in [10]. In this paper are shown significant graphs of measured physical quantities at different operating conditions of the machine; for example, it is clearly reported the influence of the valve setting at the compressor outlet on the flow velocities in the diffusers of different compressor stages, at a fixed value of rotating speed of the machine. The authors performed measurements both with and without a downstream plenum. One of the most interesting experimental diagrams in [10] is the one which reports amplitude and average frequency of surge cycles, at different valve settings, for a fixed speed of rotation, coupling the machine with a downstream volume.

\subsection{Analytical models and Active Control}

Starting from experimental observations, Greitzer and Moore developed a lumped parameters analytical model, which is able to capture transient response and therefore can perform surge analysis on the whole plant [11]. Greitzer's definitive work on surge can be regarded as the natural conclusion of the process of rigorously describing this phenomenon. In details, like all previous analytical models, it uses lumped parameters in order to be able to summarize the influence of all the essential components in the compression system (e.g. compressor, ducts, downstream volume and throttle valve). The author made use of a lumped parameter model with four equations to quantitatively describe the dynamic response of the system and defined the B parameter, which is still used to predict if a certain compressor will stall or surge, depending on the fluid dynamic circuit in which it will be placed. The meaning of this physical quantity can be interpreted as ratio between pressure forces and inertial ones and it reveals that if the first ones are larger, then the machine will experiment pressure and mass flow rate oscillations (i.e. surge); otherwise, if the second ones are higher, the flow within the machine will be subjected only to rotating stall phenomenon.

After some years, Moore performed an analytical study [12] about linear and non-linear hypothesises for the rigorous solution of the set of differential equations which govern the problem. This was made to formally prove the existence of flow disturbances which can develop in circumferential and axial direction within the machine. In this work, the hypothesis of an axisymmetric trend in pressure ratio characteristic's curve was introduced. The theoretical results obtained by the author agreed with experimental evidences about stall cell rotating velocity and highlighted the variables leading to hysteretic behaviour which is always encountered in experimental tests about rotating stall. This analytical study also proves that, under non-restrictive hypotheses, that don't correspond to any particular operating point of the machine, little disturbances of the flow may be found even prior to stall conditions, as can be easily observed in measurements of any fluid-dynamic quantity within the compressor. The final synthesis of these two masterpieces of the subject was carried out in [13] and [14]. In these publications, the authors presented their shared vision of stall and surge in multi-stage compressors: making use of the idea of axisymmetric characteristic with cubic centre section for the (non-dimensional total-to-static) pressure ratio, which means that the reverse flow region (second quadrant in the pressure ratio-mass flow rate diagram) is specular to the first quadrant one with respect to the pressure ratio (positive) axis, transients in compression systems were represented by combining a twodimensional model of the unsteady flow within the only machine and a one-dimensional 
representation of the whole compression system. The underlying equations depend from the B parameter as a system status variable and theoretical solutions obtained from them are able to reveal both pure rotating stall conditions and pure surge ones, and even combinations of the two phenomena, being thus capable of describing all the possible intermediate operative conditions which can be easily observed in experimental tests.

Starting from theory developed in ' $80 \mathrm{~s}$, some authors thought it was possible to achieve active control of stall and surge. The first paper in such direction can be considered to be [15]. After this publication, experimental work on active control became relevant, particularly because in [16] the authors demonstrated that a totally analytical prevision revealed itself to be actual in physical reality. In [17] and [18] other significant experiments on centrifugal compressors were performed, but the most relevant publication was [19]. In this work, achieved on a single-stage axial compressor, the authors succeeded in increasing stall margin, modifying the first three circumferential modes (i.e. a particular kind of circumferential disturbances).

A parallel research about active control was going on in UK, using a low speed axial compressor: this activity is reported in [20]. In this case, the author exploited a circumferential disposition of quick air jets to control the first circumferential mode and succeeded in improving stall margin. After the work of these pioneers, research on active control extended also to industrial world (e.g. Rolls-Royce), but then rapidly ended both in UK and in the United States [21].

\section{Signal processing techniques for surge detection}

An innovative approach to this issue, compared to the classical operating way described so far, is the one which concerns signal processing and vibroacoustic analysis of acquired physical signals. It deals with software operations to prevent (diagnostics) and possibly avoid (control) instability phenomena of compression systems by directly exploiting information contained in the acquired signals. This is a relevant step compared to what was done in the past, when the pioneers were not helped by digital technology and therefore could not rely on pre and post processing methods, applied to discretized data stored in computers.

Remarkable examples of acoustic and vibrational analyses for surge precursors detection on multi-stage compressors can be found in [21] and [22]. These techniques take advantage of FFT (Fast Fourier Transform) algorithms, performed on vibration and acoustical noise signals, respectively coming from accelerometers and microphones, positioned at strategic points of the compressor, to maximize information on the current state of the system coming from its response. If some peaks of vibrational amplitude at some frequencies are present, this can be regarded as an important discriminating factor. Moreover, acoustic analysis of the system might reveal the presence of critical working conditions of the compressor, even if this kind of response can be often decoupled from the vibrational one. Passing from temporal domain to the frequency one, FFT analyses can reveal disturbances appearance, exploiting the information conveyed by signal energy. This is a key aspect, which allows to go further of what the signal itself, even filtered from measurement noise, reveals, and to extrapolate modulated frequencies that cannot be seen in the first order analysis (i.e. in the signal itself). This kind of investigation, which looks for hidden periodicity contained in signals, exploiting information obtainable from higher order analyses (e.g. second order analysis deals with signal energy) is related to cyclostationary techniques, as shown in [23]. This innovative data analysis technique was performed for the first time in this field of research in [21]. The authors succeeded in demonstrating its effectiveness also in this contest, after it was proved to be efficient in other application fields, like in rolling bearings diagnostics [24]. To all effects, cyclostationary investigation 
is an example of higher order statistics analysis, which can even be extended to higher orders, if necessary; a signal is said to be cyclostationary of a certain order, if it shows a periodic trend in someone of its characterizing physical quantities (e.g. the first one is the signal itself, the second one is its energy and so on) as stated in [23]. Thus, a periodic signal is cyclostationary of first order, while a non-periodic signal can show a second order cyclostationary behaviour, which is the most common case in complex coupled systems; it can reveal the presence of "hidden periodicities "and therefore of deterministic components somehow covered by the signal itself. This can be a formidable source of information on what is actually happening inside the machine, since it is able to reveal the presence of frequencies related to rotating stall patterns, as explained in [21].

\section{Relating mechanical behaviour and fluid-dynamic instabilities}

The attempt of corelating mechanical behaviour of a compressor with its flow instabilities represents an innovative approach to stall and surge detection, based on the coupled behaviour of the system; indeed, this implies axial forces acting during surge and a change in the rotodynamic response of compressors during rotating stall. These two phenomena are respectively exploited in [6] and [7] to obtain information about the fluid-dynamic behaviour of centrifugal compressors. In [6] the authors performed surge force measurements on a centrifugal compressor operating in surge conditions, both in a direct way and deriving them from displacements measures. In the first approach, by means of load cells, they directly measured axial force intensity and related them to an average surge frequency, showing the dependence of these two variables from the downstream piping volume. In the second approach, they followed an undirect procedure to obtain surge axial forces from integration of axial vibrations measurements, with the strong hypothesis of considering thrust bearing stiffness independent from its displacements (i.e. linear modelling of bearing stiffness).

In [7] the authors obtained a diagnostic support model, starting from measuring the rotodynamic response of the system. This predictive model of the investigated centrifugal compressor is based on Finite Element Method (FEM) approach and it was proved to be a robust tool for detecting rotating stall onset. This paper is based on a previous experimental activity, in which pressure trends along circumferential direction were acquired and then appropriately post processed. As shown in [25], circumferential pressure distribution due to rotating stall is calculated and then it is used as input for calibrating the rotodynamic model: the found results are interesting and give to this kind of research interesting prospects, which are by now carried on not only by fluid-dynamic calculations, but also by structural ones. Here follows a brief explanation of the procedure made up by the authors: starting from the anomalous pressure distribution obtained due to rotating stall, a value for the stall force is evaluated by integrating this circumferential distribution on the surface on which it is imagined to be acting. After performing this calculation, this stall force is introduced in the rotodynamic model as the external load that stresses the mechanical system; this leads to a forced response to an external force that is not synchronous with the angular velocity of the machine (which is different from the classical analysis of rotor unbalance). This aerodynamic force is thought as a rotating excitation with constant modulus and frequency. Numerical FRF (Frequency Response Function) obtained from this numerical tool in a particular location of the model was finally compared with the experimental signal acquired in the same position by means of an accelerometer, showing a good agreement in terms of vibration amplitudes. The next step of the activity undertook by the authors will be the application of the model on experimental data recorded on real compressors, since by the moment the only validation has been conducted basing on scaling criterion of a real machine; the first results obtained in that direction yield however to a good predictability of 
the vibration amplitude, with a good sensibility response depending on the chosen solution for hydrodynamic bearings of the rotor (e.g. conventional hydrodynamic oil bearings versus Squeeze Film Dampers ones).

\section{Conclusions}

An overview about rotating stall and surge and their issues has been given in this paper. In particular, attention and care has been paid to the first analogical experimental works which describe these two related phenomena and the attempts to formalize in analytical way their governing physics. This gave energy to some authors to enterprise studies on active systems to perform control of stability of compression systems, enhancing their stability working field. Then, digital signal processing techniques and their potential application in this subject were highlighted. Moreover, correlations between rotodynamic response and aerodynamic blade load distribution due to rotating stall cells were underlined, and the same was made for axial forces and vibrations acting in surge events and their fluiddynamic unsteady loads. Finally, a suitable perspective might be the application of Machine Learning techniques, Supervised or Unsupervised, to compressors. In this way, treating all the energy system like a black box, one tries to exploit big data analysis techniques, to teach the system which are the stable operating points. The whole system could be able to understand when it is going to work towards an unstable region of its operating range (diagnostics); in this case an action should be done by the control system to bring back the working point to a safe operational zone (control).

This project has received funding from the European Union's Horizon 2020 research and innovation programme under grant agreement No 641073, Bio-HyPP project (http://www.bio-hypp.eu).

\section{References}

1. I. Rossi, A. Sorce, A. Traverso, Gas turbine combined cycle start-up and stress evaluation: A simplified dynamic approach, App. Energy, 190, 880-890 (2017)

2. A. Cuneo, V. Zaccaria, D. Tucker, A. Sorce, Gas turbine size optimization in a hybrid system considering SOFC degradation, App. Energy, 230, 855-864 (2018)

3. E. Munari, M. Morini, M. Pinelli, P.R. Spina, A. Suman, Experimental Investigation of Stall and Surge in a Multistage Compressor, J. Eng. Gas Turbines Power, 139, 6 (2017)

4. M. Huppert, W. Benser, Some Stall and Surge Phenomena in Axial-Flow Compressors, J. of Aeronautical Science (1953)

5. M. L. Ferrari, P. Silvestri, F. Reggio, A. F. Massardo, Surge prevention for gas turbines connected with large volume size: experimental demonstration with a microturbine, App. Energy, 230, 1057-1064 (2018)

6. K. Brun, S. Simons, R. Kurtz, E. Munari, M. Morini, M. Pinelli, Measurement and Prediction of Centrifugal Compressor Axial Forces During Surge-Part I: Surge Force Measurements, J. Eng. Gast Turbines Power, 140 (2018)

7. D. Biliotti, A. Bianchini, G. Vannini, E. Belardini, M. Giachi, L. Tapinassi, L. Ferrari, G. Ferrara, Analysis of the Rotordynamic Response of a Centrifugal Compressor Subject to Aerodynamic Loads Due to Rotating Stall, J. Turbomach., 137 (2015)

8. I. J. Day, stall, surge and 75 years of research, Proceedings of ASME Turbo Expo, Montréal, Canada, 3-8 (2015)

9. R. Bullock, H. Finger, Compressor surge investigated by NACA, SAE J., 59 (1951) 
10. G. L. Arnulfi, P. Giannattasio, C. Giusto, A. F. Massardo, D. Micheli, P. Pinamonti, Multistage Centrifugal Compressor Surge Analysis, J. Turbomach., 121, 305-311 (1999)

11. E. Greitzer, Surge and rotating stall in axial compressors, J. Eng. Gas Turbines Power, 98, 2 (1976)

12. F. Moore, A theory of rotating stall of multistage axial compressors, J. Eng. Gast Turbines Power, 106 (1984)

13. F. Moore, E. Greitzer, A theory of post-stall transients in axial compression systems: Part i - Development of equations, J. Eng. Gast Turbines Power, 108, 1 (1986)

14. E. Greitzer, F. Moore, A theory of post-stall transients in axial compression systems: Part ii - Application, J. Eng. Gast Turbines Power, 108, 2 (1986)

15. A. Epstein, J. Ffowcs Williams, E. Greitzer, Active suppression of aerodynamic instabilities in turbomachines, J. Propulsion and Power, 5, 2 (1989)

16. N. McDougall, N. Cumpsty, T. Haynes, Stall inception in axial compressors, J. Turbomach., 112, 1 (1990)

17. J. Pinsley, G. Guenette, A. Epstein, E. Greitzer, Active stabilization of centrifugal compressor surge, J. Turbomach., 113, 4 (1991)

18. D. L. Gysling, J. Dugundji, E. M. Greitzer, A. H. Epstein, Dynamic control of centrifugal compressor surge using tailored structures, J. Turbomach., 113, 4 (1991)

19. J. Paduano, A. Epstein, A. Valavani, J. Longley, E. Greitzer, G. Guenette, Active control of rotating stall in a low speed axial compressor, J. Turbomach., 115, 1 (1993)

20. I. Day, Active suppression of rotating stall and surge in axial flow compressors, J. Turbomach., 115, 1 (1993)

21. E. Munari, G. D’Elia, M. Morini, E. Mucchi, M. Pinelli, P.R. Spina, Experimental Investigation of Vibrational and Acoustic Phenomena for Detecting the Stall and Surge of a Multistage Compressor, J. Eng. Gast Turbines Power, 140 (2018)

22. M. Morini, M. Pinelli, M. Venturini, Acoustic and Vibrational Analyses on a Multistage Compressor for Unstable Behavior Precursor Identification, Proceedings of ASME Turbo Expo, Montréal, Canada (2007)

23. J. Antoni, Cyclostationary by examples, Mech. Syst. Signal Process., 23, 987-1036 (2009)

24. G. D'Elia, M. Cocconcelli, E. Mucchi, G. Dalpiaz, Combining Blind Separation and Cyclostationary Techniques for Monitoring Distributed Wear in Gearbox Rolling Bearings, J. Mech. Eng. Sci., 231, 1113-1128 (2016)

25. A. Bianchini, D. Biliotti, G. Ferrara, L. Ferrari, E. Belardini, M. Giachi, L. Tapinassi, G. Vannini, A Systematic Approach to Estimate the Impact of the Aerodynamic Force Induced by Rotating Stall in a Vaneless Diffuser on the Rotordynamic Behavior of Centrifugal Compressors, J. Eng. Gast Turbines Power, 135 (2013) 